\title{
Laser-Cooled Neutral Atom Frequency Standards
}

\author{
STEVEN L. ROLSTON AND WILLIAM D. PHILLIPS \\ Invited Paper
}

\begin{abstract}
Recent advances in laser cooling, including temperatures as low a few microkelvin, and the demonstration of the ability to launch atoms while maintaining these low temperatures, now allows the realization of atomic fountain frequency standards. An analysis of a Cs microwave fountain predicts potential stability and accuracy improvements of a factor of 100 over present laboratory standards, with an ultimate accuracy near $10^{-16}$. An optical frequency standard based on a two-photon transition in xenon is analyzed and shown to have potential stability 5 orders of magnitude better than current Cs standards, and a possible accuracy of $10^{-18}$. Neutral atom frequency standards will have much better signal-to-noise $(S / N)$ ratios than ion standards, but suffer from larger systematic effects, making the two technologies complementary in many ways.
\end{abstract}

\section{INTRODUCTION}

The motion of atoms is one of the major sources of imprecision and inaccuracy in atomic frequency standards. This has been one of the primary motivations for the development of the field of laser cooling of atoms. Trapped ion frequency standards (reviewed elsewhere in this issue by W. Itano [1]) have already been successfully demonstrated. Here we will consider the prospects for laser-cooled, neutral atom frequency standards.

One difference between laser cooling of neutral atoms and of ions is that there are no deep traps for neutral atoms, so they must be cooled while in flight, typically in an atomic beam. They can then be further cooled, and confined in optical molasses or a shallow trap. Since the neutral atoms cannot be trapped by virtue of a net charge, as are ions, traps for neutral atoms must perturb the internal energy levels of the atoms by an amount on the order of the trap depth. This perturbation of the energy levels is usually unacceptable for a frequency standard, so most proposed neutral atom standards use free atoms, possibly released from a trap. A number of authors [2], [3] have considered

\footnotetext{
Manuscript received January 9, 1991; revised March 18, 1991. This work was supported in part by the Office of Naval Research, Arlington, VA.

The authors are with the Atomic Physics Division, Physics Laboratory, National Institute of Standards and Technology, Gaithersburg, MD 20899.
}

IEEE Log Number 9101208 trapped neutral atom standards, but it appears that it will be difficult to reach the same expected performance as freeatom standards, due to shifts and broadenings associated with the interactions with the trap. In this paper, we will only consider neutral atom standards based on atoms in free flight.

Free neutral atom frequency standards cannot have the nearly infinite observation time available to trapped ion standards, so the linewidth of the clock resonance in a neutral standard will not generally be as narrow as in a trapped ion standard. On the other hand, ion standards generally operate with small numbers of ions to reduce the effects of ion-ion interactions on the motion of the ions and the performance of the frequency standard. Neutral atom standards may have significantly better signal-to-noise $(S / N)$ performance, since they are not limited to a few atoms. Recent advances in the field of laser cooling now allow interaction times of the order of one second for free atoms, opening up the possibility of narrow linewidth $(\mathrm{Hz})$ neutral atom frequency standards with better $S / N$.

\section{ADVANCES IN LASER COOLING}

Early analysis of laser cooling [4] was based on a Doppler-induced velocity dependence of the photon scattering rate, and hence the radiation pressure. This implied a "Doppler-cooling limit" with a minimum kinetic energy equal to the energy half-width at half-maximum of the cooling transition

$$
k_{B} T_{D}=\hbar \Gamma / 2
$$

a few hundred microkelvin for a typical allowed transition. In Na, the Doppler-cooling limit is $240 \mu \mathrm{K}$, corresponding to an $\mathrm{rms}$ velocity in one dimension of $30 \mathrm{~cm} / \mathrm{s}$, while in Cs the temperature limit and rms velocity are $125 \mu \mathrm{K}$, and $9 \mathrm{~cm} / \mathrm{s}$, respectively.

The recent discovery of temperatures significantly below the Doppler limit in a number of atomic systems [5], [6] enhances the possibility of neutral atom frequency standards. A theoretical understanding of the low tempera-

U.S. Government Work Not Protected by U.S. Copyright 
tures [7] is based on the multilevel nature of the atoms, involving different light shifts between the ground states and spatially dependent optical pumping between these states. The measured temperatures, $25 \mu \mathrm{K}$ in $\mathrm{Na}$ [8] (an rms velocity of $10 \mathrm{~cm} / \mathrm{s}$ ) and $2.5 \mu \mathrm{K}$ in Cs [6] (an rms velocity of $1.3 \mathrm{~cm} / \mathrm{s}$ ) correspond to atoms with a mean thermal velocity three to four times the recoil velocity, $v_{R}=\hbar k / m$, which is $3 \mathrm{~cm} / \mathrm{s}$ in $\mathrm{Na}$ and $0.35 \mathrm{~cm} / \mathrm{s}$ in Cs. It is reasonable to expect to be able to cool an atom for frequency standard applications to about three recoil velocities (or about 10 times the recoil energy) if it has a multilevel ground state. This is also approximately found in a recent theoretical treatment [9]. It should be noted that atoms with a ${ }^{1} S_{0}$ ground state such as the alkaline earths, will not experience the sub-Doppler-limit cooling.

\section{Molasses AND Magneto-Optical TRAPS (MOT's)}

In the experiments that demonstrated the lowest temperatures, the atoms were laser cooled in optical molasses, a region of three pairs of orthogonal counterpropagating laser beams detuned below the atomic resonance. This produces a viscous confinement of the atoms with a diffusion time on the order of a second, and a density of about $10^{8} \mathrm{~cm}^{-3}$ in a volume of $1 \mathrm{~cm}^{3}$. The velocity capture range for molasses requires it to be loaded from an atomic beam that is decelerated using radiation pressure from a counterpropagating laser. As the atoms decelerate, the laser detuning must change to compensate for the changing Doppler shift. This has been accomplished in two different ways: changing the "detuning" of the atom by applying a magnetic field that Zeeman shifts the resonant frequency, decreasing as the atoms slow down [10], or rapidly sweeping (chirping) the frequency of the laser to stay in resonance with the decelerating atoms [11]. In either method an atom at typical thermal oven temperatures must scatter about $5 \times 10^{4}$ photons to be stopped. The minimum distance required for stopping is

$$
r_{\min }=v_{0}^{2} \tau / v_{R}
$$

where $v_{0}$ is the initial velocity and $\tau$ is the excited state lifetime. The minimum distance, typically of order 0.5 meters, is achieved only for high saturation. To compress as well as decelerate the velocity distribution the saturation must be reduced, with the laser frequency slightly below resonance with the decelerating atoms. This increases the deceleration distance, so one meter is a more typical length for a deceleration apparatus.

The lowest temperatures in molasses can only be achieved with very low laser intensities and large laser detunings [6], [8]. This is a regime where molasses is not very effective at capturing fast atoms, although it can still cool and confine slow atoms. For this reason, it is preferable to load molasses at high laser intensity and relatively small detunings and quickly reduce the intensity and increase the detuning for about one millisecond (time enough for equilibration) to produce the lowest temperatures with large densities and number of atoms for use in a frequency standard.
The development of the magneto-optical trap (MOT) [12] now provides a more efficient way to load large numbers of cold atoms. This trap shares many similarities with optical molasses, but is a true trap with a restoring force. This is provided by a Zeeman-tuned radiation pressure force introduced with a magnetic field that increases in all directions from the center of the trap. The MOT has a larger velocity capture range than optical molasses and in some cases is able to capture directly from a thermal beam [13] and from vapor in a cell [14], either of which would allow a simplification by removing the necessity for the relatively large deceleration region. The loading of a MOT is particularly effective when the MOT is combined with a Zeeman-tuned deceleration apparatus. The temperatures achieved in a MOT are generally not as low as those in molasses [15], but the trap can be turned off, and molasses with appropriate detuning and intensity turned on for a short period of time [17], as stated previously. This produces a small (of order one millimeter diameter) dense $\left(10^{10}-10^{11}\right.$ $\mathrm{cm}^{-3}$ ) ultracold sample of atoms for use in a frequency standard.

In either case, whether the cold sample of atoms is prepared with molasses or a MOT, we can expect a final cooling period of pure optical molasses, optimized to produce the lowest possible temperatures and expected to be about 10 times the recoil energy. In a one second loading period, we assume an accumulation of about $10^{8}$ atoms in a $1 \mathrm{~cm}^{3}$ volume with molasses, or a $10^{-2} \mathrm{~cm}^{3}$ volume with a MOT.

\section{THE ATOMIC FOUNTAIN}

In this paper, we will only consider frequency standards operating on a transition with a narrow enough natural linewidth (of order one $\mathrm{Hz}$ ) that will benefit from the long interaction time possible with an atomic fountain. The atomic fountain was first proposed and attempted by Zacharias [18] and has been studied by other authors [19]. Atoms are launched upward, and pass twice through the same interaction region (a microwave or optical cavity), once on the way up and once on the way down. This constitutes the two interactions separated in time to produce Ramsey fringes [20], with a resolution determined by the time between the two interactions. This can be a very powerful technique, because the interaction time can be very long (a $30-\mathrm{cm}$ fountain gives a $0.5-\mathrm{s}$ interaction time) in a relatively compact apparatus. This may be compared to $10-20 \mathrm{~ms}$ interaction times achieved in laboratory Cs microwave standards. The Zacharias experiments were not successful because the traditional atomic beam source used did not have a sufficient flux of slow atoms. Laser-cooled sources can provide the necessary flux.

The fountain geometry has an additional advantage in that the two Ramsey interactions occur in the same cavity, which will help minimize some sources of error. Such a fountain, in which the two interactions come solely from the ballistic motion, has just been achieved by the laser cooling group at the Ecole Normale Supérieure in Paris 
(to be published in Europhys. Lett. by Clairon et al.). A similar experiment was previously performed at Stanford [17]: laser-cooled $\mathrm{Na}$ atoms were launched upwards into a cavity, where they were subjected to two microwave pulses as they rose and fell while in the cavity, and a narrow Ramsey spectrum was observed. In an experiment at the University of Colorado [21], a Ramsey resonance using pulsed microwaves was observed with free-falling laser-cooled Cs atoms.

An important consideration for a fountain is that the launch does not introduce any significant heating. In the initial Stanford experiment [17], the atoms were launched with radiation pressure from an unopposed laser beam. This produces significant heating due to the random nature of the absorptions and emissions of photons. If instead the atoms are launched by producing a moving standing wave, such that the atoms remain cold in the rest frame of the standing wave, this extra heating is not present [8], [22]. This procedure has been demonstrated at JILA in Boulder [23], at Stanford [24], and at ENS in Paris [25]. If the frequencies of the vertical pair of molasses beams is shifted, with the upward beam upshifted by $\Delta \nu$ and the downward beam downshifted by $\Delta \nu$, this creates a "moving standing wave" rising at a velocity $\lambda \Delta \nu$. The atoms in the molasses will equilibrate with this moving wave, and have zero mean velocity in the moving frame. The molasses beams can be quickly switched off, leaving the entire cloud of atoms moving upward without any significant increase in the temperature. This is the procedure used in the ENS experiments: Fig. 1 shows time-of-flight spectra for molasses-cooled Cs atoms as they fell through a detection region placed $7 \mathrm{~cm}$ under the molasses. Figure 1(a) shows a flight time of $120 \mathrm{~ms}$ for atoms dropped from molasses, while Fig. 1(b) shows a flight time of $350 \mathrm{~ms}$ for atoms that were first launched $12 \mathrm{~cm}$ upwards with such a technique. The temperature determined from this spectrum shows that to within $1 \mu \mathrm{K}$, the temperature is unchanged by the launch.

\section{A Cesium Microwave Clock}

It is fortunate that the definition of the second is based on an atom, Cs, that is ideal for laser cooling. We can now consider the expected performance of a Cs microwave standard at $9 \mathrm{GHz}$ based on a laser-cooled atomic fountain [26]. We will assume that we start with a sample of $10^{8}$ atoms having an rms velocity of $1.3 \mathrm{~cm} / \mathrm{s}$ along any one axis. A launch velocity of $2.5 \mathrm{~m} / \mathrm{s}$ (corresponding to a $3-\mathrm{MHz}$ frequency shift in the vertical molasses beams) will produce a $30-\mathrm{cm}$ fountain, with an interaction time, $T=0.5 \mathrm{~s}$. For a monoenergetic beam, this will produce a Ramsey fringe with width (FWHM) of

$$
\Delta \nu=\frac{1}{2 \mathrm{~T}}
$$

about one $\mathrm{Hz}$ in this example. The atoms will spread by about $0.7 \mathrm{~cm}$ during their flight, less than a half wavelength of the $9 \mathrm{GHz}$ microwave radiation. A high-finesse FabryPerot-type microwave cavity will allow passage of a sample of atoms of this size. The molasses puts nearly all the atoms

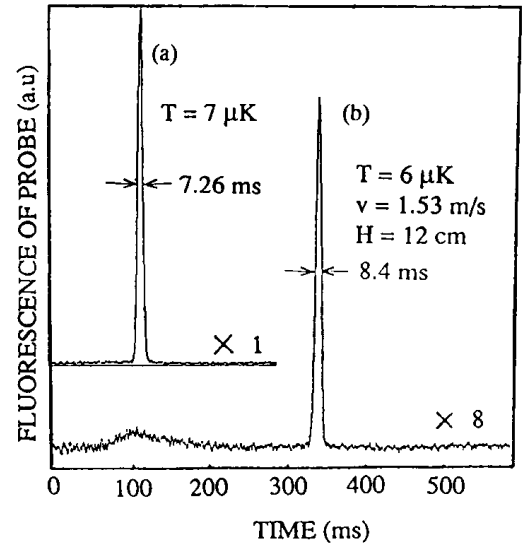

Fig. 1. Time-of-flight spectra for $\mathrm{Cs}$ atoms released from molasses at ENS. (a) Spectrum for atoms dropped from molasses. (b) Spectrum of atoms after being launched $12 \mathrm{~cm}$ upwards. Note the arrival time has increased by $230 \mathrm{~ms}$ over the previous spectrum.

into the $F=4$ ground hyperfine state, with a nominally uniform distribution of $m$ states, so we assume that $1 / 9$ of the atoms launched are in the $F=4, m_{F}=0$ state necessary for the clock transition. Allowing for spreading of atoms and the time for loading a sample, we can expect about $10^{6}$ atoms per second to make the hyperfine transition at the peak of the Ramsey spectrum. Since there is no laser light on during the flight of the atoms through the fountain, there are no light shifts that need to be considered. The atoms can be detected with essentially unit quantum efficiency by making use of the laser-cooling optical transition, which allows each atom to scatter many photons, overcoming the inherently low quantum efficiency of photon detection. This would lead to a $S / N$ limited by the shot noise due to the number of atoms making the transition when the clock is locked by sampling the sides of the central resonance. This assumes the number of atoms launched in the $F=4, m_{F}=0$ ground state in each cycle is constant, and can be approximately realized, especially if all the lasers are intensity and amplitude stabilized. In practice, it will probably be necessary to measure the total number of atoms in the correct state that were launched with an additional unit quantum efficient optical detection scheme. Assuming the shot noise limit gives a $S / N$ ratio (determined by the square root of the number of atoms) of 1000 in one second. A measure of the performance of such a standard is given by the square root of the Allan variance [27],

$$
\begin{aligned}
\sigma(\tau) & =\kappa \Delta \nu /\left[\pi \nu(S / N)(\tau / t)^{\frac{1}{2}}\right] \\
& =\kappa /\left[2 \pi \nu(S / N)(\tau T)^{1 / 2}\right]
\end{aligned}
$$

where $S / N$ is the signal-to-noise ratio for one fountain cycle, $t$ is the time between fountain cycles, $\kappa$ is a constant of order unity dependent on the details of the modulation technique used, and the second equation uses the result of (3) and assumes $t=T$, the fountain interaction time. For 
our case this gives $\sigma(\tau)=3.5 \times 10^{-14}$ at one second, and nearly $10^{-16}$ at one day. Present day laboratory Cs standards [28] have $\sigma(\tau)=1-5 \times 1^{-12}$ at one second, so the atomic fountain Cs standard represents almost a two order of magnitude improvement in stability over present day standards. To use this improved stability will require a "flywheel oscillator"-an oscillator that can maintain the phase of the oscillation during the one second update time of the fountain. This has traditionally been done with quartz oscillators, which are sufficient for current standards. The improved stability of the fountain requires a better oscillator than the best currently available quartz oscillators, which have stabilities of $1-3 \times 10^{-13}$ at one second [29]. It is likely that advances in such oscillators will continue during the time that the fountain standard is developed. Another possibility is a cryogenic hydrogen maser, which would have sufficient stability [30], although this would be a considerable complication to the standard.

The other important issue for a clock besides its stability is the accuracy. Present day Cs standards' accuracy limitations are mainly related to the atomic motion. The quoted accuracies of the primary standards around the world vary from $10^{-13}$ to $10^{-14}$ [31]. In a laser-cooled atomic fountain, the second-order Doppler shift and gravitational red shift are reduced to $10^{-17}$, with the uncertainty in the shift at the $10^{-20}$ level. The single microwave cavity eliminates end-to-end phase shift errors due to unequal electrical lengths between the microwave source and the two cavities used in a traditional standard. The primary source of inaccuracy in present standards generally arises from the distributed cavity phase shift, which is produced by losses in the cavity creating an imbalance between the power in the counterpropagating microwaves. The frequency shift due to the distributed cavity phase shift is proportional to the linewidth and will be smaller for a fountain standard than for present standards, all other things being equal. In a traditional standard, this shift reverses on reversal of the atomic beam direction and its uncertainty is limited by the ability to assure identical paths for the forward and reversed beams. In the atomic fountain, each atom reverses direction, but an exact retrace of its trajectory through the cavity probably cannot be done as well as in current standards. However, it may be possible to evaluate the distributed cavity phase shift by reversing the direction from which the microwaves are injected into the cavity. In addition, since there is only a single cavity, it should be possible to use a higher $Q$ cavity, which will reduce the size of the phase shift. Because the atoms remain localized to less than a microwave wavelength, it will also be possible to pulse the microwaves so that the atoms only interact when they are in the center of the cavity, where the wavefronts are flattest.

Stray magnetic fields become easier to deal with in a fountain since the size of the apparatus is appreciably smaller than a standard such as the 3.7-meter long NBS-6. It should be possible to reduce stray fields to better than $5 n T$, which corresponds to an uncertainty of less than $10^{-16}$ in the fountain standard. It is possible that the re- quirements for magnetic stability needed to achieve $10^{-16}$ accuracies will preclude the use of a MOT. Even if the magnetic fields of the MOT are switched off before a launch, there remains the problems of residual fields and hysteresis in the apparatus and magnetic shields that may prevent the MOT from being the source for an ultimate microwave standard.

There will be additional systematic shifts besides the few we have discussed above. The ability to evaluate such systematic shifts in a standard is limited by the statistical uncertainty (it is difficult to adjust a parameter and to evaluate the impact on the accuracy at a level below the stability achieved in a reasonable period of time-such as one day). The substantial increase in stability offered by the Cs fountain should facilitate a similar improvement in the analysis of the accuracy.

Microwave ion standards have been demonstrated [1] and have the potential for similar long-time performance. Since a neutral atom standard typically would operate with larger numbers of atoms than an ion standard, it would have better $S / N$, leading to better short term stability according to (4). Because an ion standard may use an indefinitely long Ramsey interaction time, its $\sigma(\tau)$ can be improved to equal that of a standard with higher $S / N$. All other things being equal, this would be achieved by increasing the interaction time $T$ by the ratio of the squares of the $S / N$ of the two standards. Comparisons between laser-cooled ion and neutral atom standards may offer a unique opportunity for an accurate evaluation of systematic errors in both systems. An atomic fountain Cs standard also offers the real possibility of a rugged, reliable, and portable standard due to its small size and the availability of diode lasers for the laser cooling. If the magnetic field problems associated with a MOT can be overcome, then an even more compact standard loaded in a vapor cell can be envisaged [26]. A frequency standard operating in near-zero gravity (not, of course, as a fountain) could take advantage of additional cooling by trap expansion [32] to achieve longer observation times.

\section{A Neutral Atom Optical Clock}

The same laser-cooling and atomic fountain techniques used in a microwave frequency standard can be applied in the optical frequency region, with the long term goal of an improved standard, due to the four to five order of magnitude higher $Q$ available in a one $\mathrm{Hz}$ optical transition. A number of different potential optical frequency standards have been considered [33], but we will restrict consideration to transitions with linewidths of order one $\mathrm{Hz}$, that can benefit from an atomic fountain. We do not consider here how to connect an optical frequency to the microwave region, a problem which has been discussed extensively, for which some new ideas are being considered, and on which much work remains to be done [34].

There are a number of requirements for an atomic fountain optical frequency standard: first of all, the atom should possess a long-lived metastable state $(\tau>0.1 \mathrm{~s})$ for the

PROCEEDINGS OF THE IEEE, VOL. 79, NO. 7, JULY 1991 
clock transition, which will produce a clock with a line- $Q$ of $10^{14}-10^{15}$ (to be contrasted to $10^{10}$ for the microwave standard). Secondly, this long-lived state should be accessible with a two-photon "allowed" transition connecting the ground state with the long-lived state (both states must have the same parity with $\Delta J=0$ or 2). An "allowed" twophoton transition is one that is mediated with two electric dipole matrix elements. If a higher order transition (such as electric quadrupole) is used, the power required to produce a reasonable two-photon transition probability will be so large that the AC Stark shift (which is produced from electric dipole matrix elements-see the following) will be much larger than the linewidth, leading to an intractable problem with accuracy due to the power-dependent shift. A two-photon transition is advantageous so that the interaction is first-order Doppler-free and interacts with all the atoms. One might think that the atoms are cold enough that a saturation spectroscopic technique might be efficient, but the Doppler width of ultracold atoms is still of order $10^{4} \mathrm{~Hz}$, so a one- $\mathrm{Hz}$ transition will only interact with $10^{-4}$ of the atoms. It may be possible to pulse the beams, such that the Fourier transform of the laser pulse covers the Doppler width, allowing interaction with all the atoms. In any case, the saturated absorption would require at least three interaction regions [35], so the lasers would have to be pulsed in any case. Thirdly, the atom must be laser-coolable: it must have an allowed cycling transition from the ground state, and it is preferable if only one laser is required (no repumpers). To take advantage of the sub-Doppler cooling mechanisms, the atom should have a degenerate ground state $\left(J_{g}>0\right)$. Finally, there needs to be an efficient method to detect whether or not the clock transition has been made.

A number of atoms have been suggested that meet some or all of these requirements (see [33] for a partial list). Most of the candidates have been based on a metastable $D$ state in the alkaline earths $\left({ }^{40} \mathrm{Ca},{ }^{88} \mathrm{Sr},{ }^{138} \mathrm{Ba}\right)$, but these atoms will be limited to Doppler-cooling temperatures due to the nondegenerate ground state. Another very promising candidate is a transition in ${ }^{109} \mathrm{Ag}$, which meets all the requirements mentioned above, except that the laser-cooling transition is in the UV at $328 \mathrm{~nm}$ where it will be difficult to obtain adequate laser power. We will offer as an example a previously overlooked candidate, metastable xenon, that actually meets all of the above requirements.

\section{Metastable Xenon}

A partial diagram of the energy levels for xenon is shown in Fig. 2. The first excited state is extremely metastable, with an estimated lifetime of $150 \mathrm{~s}$ [36]. For the purpose of the clock we will consider this ${ }^{3} P_{2}$ state to be the ground state. Laser cooling is very favorable with a cycling transition at $882 \mathrm{~nm}$ (ideal for a Titanium:sapphire laser) with a 30-ns lifetime. The relevant laser cooling atomic parameters for metastable noble gases are essentially those of the neighboring alkali. In the case of xenon, this means it acts essentially like cesium, with similar stopping pa-

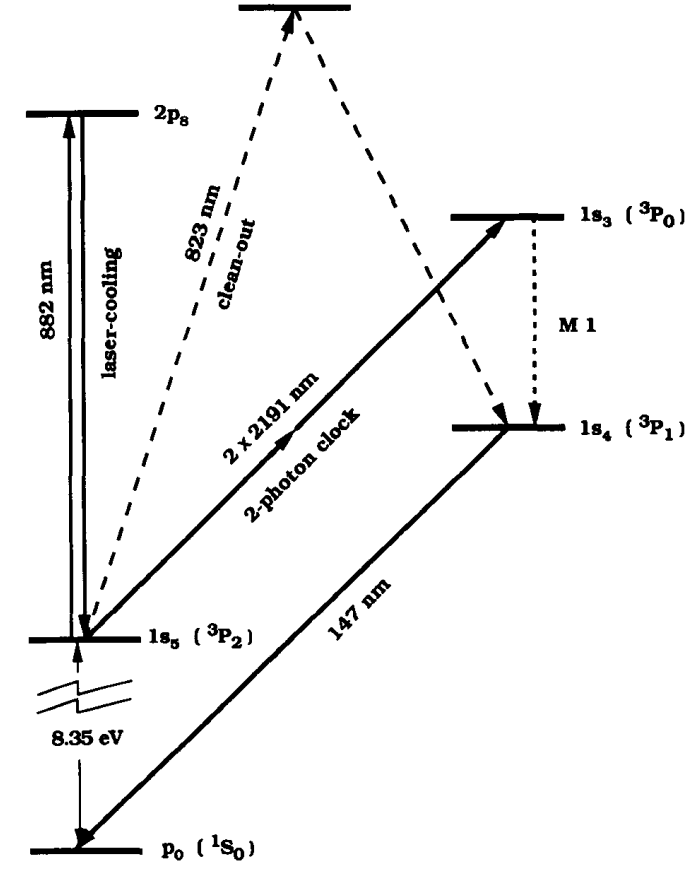

Fig. 2. Energy levels and relevant transitions in xenon (not to scale).

rameters, Doppler-cooling limits, and sub-Doppler cooling limits. Note that xenon has both odd and even isotopes: we can choose an even isotope with nuclear spin $I=0$, so there will be no hyperfine structure to complicate the cooling process, although the degenerate $J=2$ state will still allow the sub-Doppler cooling mechanisms to be active. In analogy with Cs, we can expect to be able to laser cool to about $2 \mu \mathrm{K}$, which corresponds to a one-dimensional rms velocity of $1.2 \mathrm{~cm} / \mathrm{s}$.

The optical clock transition (see Fig. 2) is a two-photon transition connecting the ${ }^{3} P_{2}$ state with the ${ }^{3} P_{0}$ state that is $9129 \mathrm{~cm}^{-1}$ higher lying. The ${ }^{3} P_{0}$ state decays to the ${ }^{3} P_{1}$ state via a magnetic dipole transition, and has a calculated lifetime of $0.08 \mathrm{~s}$ [36], which corresponds to a linewidth of $2 \mathrm{~Hz}$. The wavelength of the radiation necessary for the two-photon transition is $2.19 \mu \mathrm{m}$. There are a number of possible sources for this radiation: a color-center laser, $\mathrm{Co}: \mathrm{MgF}_{2}$ laser, a $\mathrm{CW}$ optical parametric oscillator based on Nd:YAG, or difference generation in a buildup cavity with appropriately chosen fundamental lasers.

The metastable ${ }^{3} P_{0}$ "clock state" has $9.5 \mathrm{eV}$ of excitation energy, which allows the possibility of metastable detection, via emission of a secondary electron from the surface after the xenon deexcites. Quantum efficiencies in the range of a few to fifty percent are possible [37]. There are many surfaces with work functions less than $9.5 \mathrm{eV}$, but it is important that the surface be kept clean of high-work function contaminants. Of course any metastable detector would also be sensitive (although somewhat less) to the lower lying metastable state (with $8.3 \mathrm{eV}$ of excitation 
energy), but this state can be completely depopulated by the application of radiation from a laser diode at $823 \mathrm{~nm}$ that drives an allowed transition (see Fig. 2) with a $26 \%$ branching rate for decay to the ${ }^{3} P_{1}$ state which decays with a 4-ns lifetime to the atomic ground state. Application of $100 \mu \mathrm{W} / \mathrm{cm}^{2}$ of this radiation for $1 \mathrm{~ms}$ will completely remove any atoms that did not make the clock transition with an approximately $10^{6}$ rejection ratio, allowing an essentially background-free method of detection.

There are a number of possibilities for optical detection: one can apply radiation at $821 \mathrm{~nm}$ to drive the atom into a state where it will decay to the ground state, and detect the VUV photon emitted; the number of atoms remaining in the ${ }^{3} P_{2}$ state (a "flop-out" technique) can be detected with essentially unit quantum efficiency utilizing the 882-nm cycling transition to scatter many photons per atom, or one can first clean-out the ${ }^{3} P_{2}$ state with the 823 -nm laser, then drive all the atoms from the ${ }^{3} P_{0}$ clock state with 764-nm radiation back to the ${ }^{3} P_{2}$ state where they can be detected with the cycling transition. For the purposes of the evaluation of this system as a frequency standard, we will assume a $10 \%$ quantum efficiency for detection of the clock transition.

A similar analysis to that of the Cs microwave clock can be made for this optical clock. Although a source of metastable atoms will have significantly lower flux than is possible for a ground state atom source, Shimizu [38] has demonstrated in neon the ability to load metastables very efficiently into a MOT to a density limited by collisions and radiation trapping. The higher frequency of an optical clock over a microwave clock makes the effect of magnetic fields less severe on a relative basis, so the use of a MOT is more attractive. If we set as a goal a long-time stability and accuracy of $10^{-18}$ for the optical clock, to contrast with $10^{-16}$ for the ultimate microwave standard, the $10^{4}$ larger $Q$ of the optical clock makes the requirements for reducing shifts from magnetic fields 100 times less stringent.

For this estimate we will assume an order of magnitude fewer atoms than the $\mathrm{Cs}$ example, $10^{7}$ atoms in a $1-\mathrm{mm}$ diameter volume, with a similar velocity of $1.2 \mathrm{~cm} / \mathrm{s}$. Since the lifetime of the upper metastable state is only about $0.1 \mathrm{~s}$, there is no advantage to be gained in constructing a fountain with a flight time much more than about twice this lifetime (the Ramsey method produces a ground-excited coherence that decays at half the rate of the excited state). In this case we will consider a $5-\mathrm{cm}$ fountain. Note that the shorter fountain, with less time for the atoms to spread out, is well suited to a MOT with its small volume, and an optical clock with a small interaction region. The stability can be estimated analogously to the Cs clock: we assume $20 \%$ of the atoms to be in the $m=0$ ground, state, $50 \%$ to be lost due to spreading and other effects, and a one second load time. Because of spontaneous emission from the ${ }^{3} P_{0}$ state, the signal will be reduced by a factor of three. We find the square root of the Allan variance to be $\sigma(\tau)=2 \times 10^{-17}$ at one second and better than $2 \times 10^{-18}$ at 100 seconds if the stability were limited by shot noise and a $10 \%$ detection efficiency. Note that this improvement over the microwave standard comes entirely from the higher frequency of operation. As in the microwave case, the stability will depend on shot-to-shot constancy in the number of atoms, which will again most likely be continuously monitored, although the requirements are less since there are fewer atoms than the Cs case. This clock will also need a flywheel oscillator-for the optical system this will be an ultrastable optical cavity. Although a cavity with the necessary millihertz stability does not currently exist, there is much effort and progress being made in this direction [39], [40].

Unfortunately, this phenomenal stability is not easily matched in accuracy in the neutral atom optical clock. The two-photon resonant Rabi frequency [41] can be written as

$$
\Omega^{(2)}=\frac{1}{2} e^{2} \hbar\left|E_{0}\right|^{2} \sum_{i} \frac{\langle f|r \cdot \hat{e}| i\rangle\langle i|r \cdot \hat{e}| g\rangle}{\omega_{g i}-\omega_{L}}
$$

where $g, i$, and $f$ denote the ground, intermediate, and final states respectively, $\omega_{L}$ is the laser frequency, and $E_{0}$ is the optical electric field. The summation extends over all intermediate states, but in practice, is usually dominated by a few states with electric-dipole allowed matrix elements. Most of the optical clock candidates do not have any nearly resonant intermediate states, so the detuning denominator is essentially an optical frequency. The requirement on intensity to obtain the maximum signal in the Ramsey technique is to produce a $\pi / 2$ pulse in each interaction, so that $2 \Omega^{(2)} t=\pi$, where $t$ is the time of one flight through the interaction region. In practice this region would be a high finesse optical cavity, which gives considerable power buildup, lessens the power requirements on the optical source, and assures a good balance between the optical waves propagating in opposite directions.

The AC Stark shift has a very similar form to the twophoton Rabi frequency, since it is essentially the difference between the energy shifts of the ground and excited states due to two-photon transitions that couple through intermediate states back to the same initial state. The AC Stark shift on resonance can be written as:

$$
\begin{aligned}
\Delta \omega_{f g}=-\frac{1}{4} e^{2} \hbar^{-2}\left|E_{0}\right|^{2} & \left(\sum_{i} \frac{\langle g|r \cdot \hat{e}| i\rangle\langle i|r \cdot \hat{e}| g\rangle}{\omega_{g i}-\omega_{L}}\right. \\
& \left.-\sum_{i} \frac{\langle f|r \cdot \hat{e}| i\rangle\langle i|r \cdot \hat{e}| f\rangle}{\omega_{g i}-\omega_{L}}\right) .
\end{aligned}
$$

Again, the dominant matrix elements are all electric dipoleallowed, roughly the same size as those in the two-photon Rabi frequency, so the Stark shift will be of comparable size unless there is strong cancellation between the shifts of the ground and excited states. If we imagine that the twophoton transition proceeds through a single intermediate state, we can write the two-photon Rabi frequency as $\Omega^{(2)}=\kappa S_{g i} S_{i f}$ where $S_{j k}$ denotes the square of the matrix element connecting states $j$ and $k$, and $\kappa$ is a 
constant. The AC Stark shift can then be written as $\Delta \omega_{f g}=$ $1 / 2 \Omega^{(2)}\left(S_{g i}^{2}-S_{i f}^{2} / S_{g i} S_{i f}=\eta \Omega^{(2)}\right)$. If both transitions are of similar size, then the constant $\eta$ will be of order unity, and the AC Stark shift will be about equal to the two-photon Rabi frequency. As was pointed out by previous authors [20], [33] most of the time the atoms are in a field free region, so they see no light field and do not suffer any shifts. An analysis of the total effective Stark shift of the Ramsey signal gives $\Delta \omega_{f g}^{(e f f)}=\Delta \omega_{f g} t / T$ where $t$ is the interaction time in the optical cavity, and $T$ is the flight time of the fountain. From this alone it appears that a small interaction region (short interaction time) is favorable (as discussed in [33]), but if we require a $\pi / 2$ pulse the Rabi frequency is inversely proportional to the interaction time, $\Omega^{(2)}=\pi / 2 t$. If we then substitute this into the expression for the effective AC Stark shift, we find that $\Delta \omega_{f g}^{(e f f)}=\eta \Omega^{(2)} t / T=\eta \pi / 2 T \cong 1 / T$. The Stark shift of the fountain is independent of the interaction time, and is of order the inverse of the fountain time, $T$. On the other hand, if we relax the $\pi / 2$-pulse condition with a subsequent loss of signal, the dependence on the pulse area means that a factor of two reduction in the area (which also reduces the AC Stark shift by a factor of two) makes only a $30 \%$ reduction in signal.

For the specific example of metastable xenon, we have performed some preliminary calculations using calculated oscillator strengths [42], and summing over six intermediate states find that for a 1-ms interaction time, we require $87 \mathrm{~W}$ of optical power (inside the buildup cavity) to produce a $\pi / 2$-pulse. This causes a $0.6 \mathrm{~Hz}$ AC Stark shift, which corresponds to a $5 \times 10^{-15}$ shift in the frequency. Control of the intensity of the radiation to $1 \%$ should lead to an instability in the shift of $5 \times 10^{-17}$ and to realize the $10^{-18}$ long term stability implied by the previous analysis will require controlling the intensity to $.02 \%$, certainly a challenge, although not an impossibility. If we choose to reduce the optical power and accept a loss in $S / N$ so that the stability reaches $10^{-18}$ in one day instead of 100 seconds, the instability due to the AC Stark shift can be reduced to the $10^{-18}$ level and only require $0.6 \%$ amplitude stabilization.

What is often the primary source of inaccuracy in a microwave standard is the distributed cavity phase shift due to intensity imbalance in the two traveling waves that form the standing wave in the cavity. This produces a residual first order Doppler shift which can be seen to arise from a phase shift across the cavity. This particular phase shift is totally absent in the two-photon optical clock since the Doppler-free two-photon transition requires the near-simultaneous absorption of two photons from opposite directions, and is insensitive to intensity imbalance in the counterpropagating beams. There is, however, an important shift due to an angular misalignment of the optical waves in the cavity (or mismatch in the wavefront curvature of the counterpropagating waves), which allows some residual first-order Doppler shift to appear.

The Doppler-free nature of the two-photon transition relies on the cancellation of the wave vectors due to their opposite directions of propagation. If the transverse velocity of the atoms in the fountain is $1.3 \mathrm{~cm} / \mathrm{s}$, the first-order Doppler-broadened line would be approximately $10 \mathrm{kHz}$ wide, which is about $10^{-10}$ of the optical frequency. To reduce this effect which will inhomogeneously broaden the line to less than the natural width will require a wave vector mismatch to be $\delta k / k<10^{-3}$. If we assume the transverse velocity distribution is symmetric to within $10 \%$, a reduction in the shift in the center frequency to less than $10^{-16}$ will require $\delta k / k<10^{-5}$. For this reason it will be important to use a high finesse optical cavity, probably in the confocal configuration. The mode of such a cavity is insensitive to mirror misalignment and should allow a reduction of the wavevector mismatch to a level much better than the inverse of the finesse of the cavity. (A simple estimate of the mismatch for a plane-plane cavity would be $\delta k / k \cong\left(w_{0} / L\right) 1 / F$, where $w_{0}$ is the mode size on the mirror, $L$ is the cavity length and $F$ is the cavity finesse. For a cavity with $F=10^{4}, \delta k / k$ could be about $10^{-6}$ and should presumably be much better for a confocal cavity which is insensitive to angular misalignment.) As with the AC Stark shift, this source of error can also be reduced at a cost of signal, in this case by collimating the launched atoms to reduce the transverse velocity. Finally, the gravitational red shift plus the secondorder Doppler shift produce a fractional shift [20] of $1 / 6$ $v_{0}^{2} / c^{2}$, which for the $5-\mathrm{cm}$ fountain we are considering would be $2 \times 10^{-18}$.

As a check of the systematic errors in the two-photon clock, it may be attractive to drive the electric quadrupole transition with a single photon (one could frequency double the two-photon radiation). The power requirements are minimal, since the saturation intensity for such a transition will be nanowatts $/ \mathrm{cm}^{2}$. As mentioned previously, it would require at least three interactions, and could be accomplished by pulsing on the radiation at the appropriate positions in the atoms' trajectories. If this is easily done (which requires great attention to phase shift problems absent in the two-photon approach), it would offer a different source of errors than the two-photon transition and might help in understanding and characterizing the sources of inaccuracy, all within the same system.

Optical frequency standards based on trapped ions have been proposed and linewidths of less than $80 \mathrm{~Hz}$ have already been observed [1]. Because the ions are in the Lamb-Dicke regime, where the photon recoil is absorbed by the essentially infinite mass of the trap, the ion standards can work with a single photon transition, and are substantially free of the problems associated with the twophoton transition: the AC Stark shift, and the residual firstorder Doppler shift from wavevector mismatch. As in the microwave case, however, they work with small numbers of ions and the $S / N$ performance will be much less than a neutral atom standard. There will be a very fruitful opportunity to compare the performance, both stability and accuracy, of these two completely different types of optical clocks. 


\section{CONCLUSION}

The recent advances in producing ultracold temperatures with laser cooling and the demonstration of the atomic fountain have opened the door for the development of a new generation of atomic frequency standards based on laser-cooled neutral atoms, both in the microwave and optical domains. A Cs microwave standard has the potential for $10^{-14}$ stability at one second and an accuracy of $10^{-16}$, both two orders of magnitude improvement over the best current standards (although Cs beam standards under development should do substantially better [43]). In the optical domain, we have presented as a good candidate a two-photon transition in metastable xenon, with a line-Q of $10^{14}$, short term stability of $10^{-17}$, and a possible ultimate accuracy of $10^{-18}$. Trade-offs between stability and accuracy are possible because of the large $S / N$ possible in neutral atom standards. These standards will be both competitive and complementary to ion-based standards, with better $S / N$, but larger sources of inaccuracy. Comparisons between these different types of standards will offer an unprecedented opportunity to understand the performance and systematics of a new generation of frequency standards.

\section{ACKNOWLEDGMENT}

The authors would like to acknowledge helpful discussions with J. L. Hall, C. Salomon, and A. Clairon. We thank R. Drullinger and W. Itano for a careful reading of the manuscript.

\section{REFERENCES}

[1] W. Itano, "Atomic ion frequency standards," Proc. IEEE, vol. 79, pp. 936-942, this issue.

[2] D. Pritchard, "Cooling neutral atoms in a magnetic trap for precision spectroscopy," Phys. Rev. Lett., vol. 51, p. 1336, 1983.

[3] D. Kleppner, "Trapped atomic hydrogen," in The Hydrogen Atom, G. Bassani, M. Inguscio, and T. Hansch, Eds. Berlin, Germany: Springer-Verlag, 1989, p. 103.

[4] J. Gordon and A. Ashkin, "Motion of atoms in a radiation trap," Phys. Rev., vol. A21, p. 1606, 1980.

[5] P. Lett, R. Watts, C. Westbrook, W. Phillips, P. Gould, and H. Metcalf, "Observation of atoms laser cooled below the Doppler limit," Phys. Rev. Lett., vol. 61, p. 169, 1988.

[6] C. Salomon, J. Dalibard, W. Phillips, A. Clairon, and S. Guelatti, "Laser cooling of cesium atoms below $3 \mu \mathrm{K}$," Europhys. Lett., vol. 12, p. 683, 1990.

[7] J. Dalibard and C. Cohen-Tannoudji, "Laser cooling below the Doppler limit by polarization gradients: Simple theoretical models," J. Opt. Soc. Amer., vol. B6, p. 2023, 1989.

[8] P. Lett, W. Phillips, S. Rolston, C. Tanner, R. Watts, and C. Westbrook, "Optical molasses," J. Opt. Soc. Amer., vol. B6, p. 2084, 1989.

[9] Y. Castin and J. Dalibard, "Quantization of atomic motion in optical molasses," Europhys. Lett., to be published.

[10] W. Phillips, J. Prodan, and H. Metcalf, "Laser cooling and electromagnetic trapping of neutral atoms," J. Opt. Soc. Amer., vol. B2, p. 1751,1985 .

[11] J. Prodan and W. Phillips, "Chirping the light-fantastic?," in Lasercooled and Trapped Atoms, W. Phillips, Ed., Nat. Bur. Standards (U.S.) Spec. Pub. 653, 137 (1983); W. Ertmer, R. Blatt, J. Hall, and M. Zhu, "Laser manipulation of atomic beam velocities: Demonstration of stopped atoms and beam reversal," Phys. Rev. Lett., vol. 54, p. $996,1985$.

[12] E. Raab, M. Prentiss, A. Cable, S. Chu, and D. Pritchard, "Trapping of neutral sodium atoms with radiation pressure," Phys. Rev. Lett., vol. 59 , p. $2631,1987$.
[13] A. Cable, M. Prentiss, and N. Bigelow, "Observations of sodium atoms in a magnetic molasses trap loaded by a continuous uncooled source," Opt. Lett., vol. 15, p. 507, 1990.

[14] C. Monroe, W. Swann, H. Robinson, and C. Wieman, "Very cold trapped atoms in a vapor cell," Phys. Rev. Lett., vol. 65, p. 1571, 1990.

[15] The reason for the relatively high temperatures in MOT's most likely has to do with the operating conditions-high laser power and small detunings. What optimizes the density does not minimize the temperature. It should be noted that a typographical error (C. Wieman, private communication) in [14] reported a temperature in a Cs MOT of $30 \mu \mathrm{K}$, when the correct value was $300 \mu \mathrm{K}$. Recent work [16] has shown that under suitable conditions it is possible to obtain sub-Doppler temperatures in a MOT.

[16] A. M. Steane, and C. Foot, "Laser cooling below the Doppler limit in a magneto-optical trap," Europhys. Lett., vol. 14, p. 231, 1991

[17] M. Kasevich, E. Riis, S. Chu, and R. DeVoe, "RF spectroscopy in an atomic fountain," Phys. Rev. Lett., vol. 63, p. 612, 1989.

[18] J. Zacharias, Phys. Rev., vol. 94, p. 751T, 1954.

[19] R. Beausoleil and T. Hansch, "Ultrahigh-resolution two-photon optical Ramsey spectroscopy of an atomic fountain," Phys. Rev., vol. A33, p. 1661, 1986.

[20] N. Ramsey, Phys. Rev., vol. 76, p. 996, 1949.

[21] C. Monroe, H. Robinson, and C. Wieman, "Observation of the cesium clock transition using laser-cooled atoms in a vapor cell," Opt. Lett., vol. 16, p. 50, 1991.

[22] P. Lett, C. Westbrook, R. Watts, S. Rolston, P. Gould, H Metcalf, and W. Phillips, "Atoms laser-cooled below the Dopplercooling limit," in Frequency Standards and Metro$\log y$, A. DeMarchi, Ed. Berlin, Germany: Springer-Verlag, 1989, p. 264 .

[23] J. Hall and M. Zhu, private communication.

[24] D. Weiss, in Proc. Light Induced Kinetic Effects, Elba, Italy 1989, to be published.

[25] C. Salomon, J. Dalibard, W. Phillips, A. Clairon, and S. Guelatti, Europhys. Lett., vol. 12, p. 683, 1991.

[26] W. Phillips, P. Lett, S. Rolston, C. Tanner, R. Watts, C. Westbrook, C. Salomon, J. Dalibard, A. Clairon, and S. Guelatti, "Atoms in optical molasses: Applications to frequency standards," in Proc. Fourth European Frequency and Time Forum, Nuechatel, Switzerland, 1990, p. 273.

[27] D. Wineland, W. Itano, J. Bergquist, J. Bollinger, F. Diedrich, and S Gilbert, "High accuracy spectroscopy of stored ions," in Frequency Standards and Metrology, A. DeMarchi, Ed. Berlin, Germany: Springer-Verlag, 1989, p. 71.

[28] J. Vanier and C. Audoin, The Quantum Physics of Atomic Frequency Standards, Adam Hilger, Bristol, U.K., 1989, vol. 2, p. 782.

[29] R. J. Besson, "RF local oscillators for future frequency standards," in Frequency Standards and Metrology, A. DeMarchi, Ed. Berlin, Germany: Springer-Verlag, 1989, p. 157, and Fred Walls, private communication, 1990

[30] R. Vessot, E. Mattison, R. Walsworth, and I. Silvera, "The cold hydrogen maser," in Frequency Standards and Metrology, A. DeMarchi, Ed. Berlin, Germany: Springer-Verlag, p. 88.

[31] C. Audoin, "A point of view on accuracy performances and on the prospects of improvement of primary cesium beam frequency standards," in Frequency Standards and Metrology, A. DeMarchi, Ed. Berlin, Germany: Springer-Verlag, p. 46.

[32] S. Chu, J. Bjorkholm, A. Ashkin, J. Gordon, and L. Hollberg, Opt. Lett., vol. 73, 1986

[33] J. Hall, M. Zhu, and P. Buch, "Prospects for using laser-prepared atomic fountains for optical frequency standards applications," $J$. Opt. Soc. Amer., vol. B6, p. 2194, 1989.

[34] H. Telle, D. Meschede, and T. Hansch, "Realization of a new concept for visible frequency division: Phase locking of harmonic and sum frequencies," Opt. Lett., vol. 15, p. 532, 1990; T. Sato, S. Singh, S. Swartz, and J. Hall in Int. Conf. Quantum Electronics Technical Digest Series 1990, Vol. 8, Optical Society of America Washington, DC, 1990 , p. 216; and D.J. Wineland, "Laser-tomicrowave frequency division using synchrotron radiation," J. Appl. Phys., vol. 50, p. 2528, 1979.

[35] J. Bergquist, S. Lee, and J. Hall, in Laser Spectroscopy III. Berlin, Germany: Springer-Verlag, 1977, p. 142.

[36] N. Small-Warren and L. Chow Chiu, "Lifetime of the metastable ${ }^{3} \mathrm{P}_{2}$ and ${ }^{3} \mathrm{P}_{0}$ state of rare-gas atoms," Phys. Rev., vol. A11, p. 1777 , 1975 .

[37] F. Dunning, R. Rundel, and R. Stebbings, Rev. Sci. Instrum., vol. 46, p. $697,1975$.

PROCEEDINGS OF THE IEEE, VOL. 79, NO. 7, JULY 1991 
[38] F. Shimizu, K. Shimizu, and H. Takuma, "Laser cooling and trapping of Ne metastable atoms," Phys. Rev., vol. A39, p. 2758, 1989.

[39] M. Raisen, F. Elsner, J. Bergquist, W. Itano, and D. Wineland in Int. Conf. Quantum Electronics Technical Digest Series 1990, Vol. 8 Optical Society of America, Washington, DC, 1990, p. 218.

[40] C. Salomon, D. Hils, and J. Hall, "Laser stabilization at the millihertz level," J. Opt. Soc. Amer., vol. B5, p. 1576, 1988.

[41] N. Bloembergen and M. Levenson, "Doppler-free two-photon absorption spectroscopy," in High-Resolution Laser Spectroscopy, K. Shimoda, Ed. Berlin, Germany: Springer-Verlag, 1976, p. 315.

[42] C.W. Clark, private communication.

[43] R. Drullinger, D. Glaze, J. Lowe, and J. Shirley, presented at CPEM'90, Ottawa, Ont., June 1990, and IEEE Trans. Instrum. Meas., vol. 40, pp. 162-164, June 1991.

Steven L. Rolston was bom in Woodbury, NJ on November 8, 1958. He received the B.A. degree from Wesleyan University, Middletown, CT, in 1980, and the Ph.D. degree in physics from the State University of New York at Stony Brook in 1986. His thesis involved high sensitivity laser spectroscopy of unstable isotopes formed at a heavy ion accelerator.

He spent two and a half years as a postdoctoral fellow, first at the University of Washington at Seattle and then at Harvard University in Cambridge, MA, working on precision mass spectroscopy in Penning traps, towards the measurement of the inertial mass of the antiproton. Since 1988 he has been at the National Institute of Standards and Technology working on laser cooling and trapping of neutral atoms, and vacuum ultraviolet generation for precision spectroscopy.

Dr. Rolston is a member of the American Physical Society.
William D. Phillips was bom in Wilkes-Barre, PA, on November 5, 1948 He received the B.S. degree from Juniata College, Huntingdon, PA, in 1970 , and the Ph.D. degree in physics from the Massachusetts Institute of Technology, Cambridge, MA, in 1976. His thesis research involved a precision measurement of the magnetic moment of the proton and a study of collisions of laser-excited atoms.

He spent two years as a postdoctoral fellow at MIT, working on spin-polarized hydrogen and continuing work on collisions and laser spectroscopy. In 1978 he joined the National Institute of Standards and Technology (then the National Bureau of Standards), working on the measurement of the fine structure constant and the realization of the ampere. More recently he has been working on the laser cooling and trapping of neutral atoms, collisions between laser-cooled atoms, and applications of cooled atoms to studies of quantum optics and frequency standards.

Dr. Phillips is a member of the Optical Society of America and a Fellow of the American Physical Society. 